\title{
CONTROVERSIAS
}




\section{PROMOCION AUTOMATICA Y FRACASO ESCOLAR EN COLOMBIA ${ }^{1}$}

Manuel V. Rojas Rubio ${ }^{2}$

\section{Introducción}

Desde la perspectiva de las sociedades modernas la escuela es una institución central dentro del proyecto modernizador. Es su misión incorporar a las nuevas generaciones dentro de la cultura escrita $-\mathrm{y}$ a través de ella- en las representaciones lógicas y axiológicas de la modernidad. Desde esta mirada la institución escolar de básica primaria existe para brindar al conjunto de los niños en edad escolar las herramientas que les permita apropiarse de los códigos lingüísticos y matemáticos y los valores fundamentales del pensamiento moderno a través del proceso de escolarización, de manera tal que, gracias a dichas herramientas, puedan participar de forma activa durante su vida adulta en los procesos culturales y sociales, en las actividades de producción, en el ingreso, y en las decisiones de la vida política.

Dentro de este contexto, la escuela deberá ser una institución generadora de equidad socioeconómica y cultural. A través de la enseñanza y la institucionalización, se espera que la escuela aporte a los sectores marginados y desfavorecidos el "capital cultural" requerido para una participación social dinámica y constructiva. De acuerdo con el proyecto modernizador, la escuela debe constituirse en una institución compensatoria del "déficit cultural moderno" requerido para una interacción menos desequilibrada de los sectores populares con los procesos económicos y políticos dominantes en el actual momento histórico, de los cuales no es posible sustraerse.

Lo anterior a nivel práctico implicaría brindar las mejores oportunidades de aprendizaje escolar a aquellos sectores poblacionales que presentan las mayores deficiencias, como condición de posibilidad para promover el desarrollo y superar la marginalidad.

Sin embargo, después de 40 años de un proceso de escolarización creciente en América Latina -y en particular en Colombia-, los resultados del mismo son bastante contradictorios frente a las expectativas generadas. Diferentes síntomas vienen señalando el fracaso de los anteriores ideales: altas tasas de analfabetismo, deserción y repitencia escolar, bajos niveles de logro académico en los aprendizajes básicos, entre otros. Lo más grave es que esta problemática se ubica precisamente en los sectores más desprotegidos de la sociedad, hacia los que supuestamente estaban orientados los mejores esfuerzos de esta propuesta.

Frente a la crisis, el sistema educativo colombiano ha venido desarrollando una serie de políticas y reformas en la Escuela Básica Primaria en la perspectiva de superarla. Dentro del conjunto de estas reformas la Promoción Automática como estrategia para enfrentar el fracaso escolar ha venido apareciendo como una de las estrategias educativas más importantes en diferentes programas [Escuela Unitaria, Escuela Nueva,

\footnotetext{
${ }^{1}$ Muchas de las ideas presentadas en este documento se desarrollaron a través de un proceso colectivo de estudio y discusión sostenido con Marina Camargo y Rodrigo Villar en el mareo del proyecto: MCGINN, N Cls [1992] "La repitencia de grado en la Escuela Básica Primaria Colombiana. —Resultados preliminaresSanta Fe de Bogotá, D.C., Ministerio de Educación Nacional, Harvard Institute for International Development Centro de Investigaciones de la Universidad Pedagógica Nacional. El autor agradece los aportes hechos por Gloria Calvo en la discusión de los borradores preliminares de este artículo.

2 Investigador del Centro de Investigaciones de la Universidad Pedagógica Nacional y de la División de Evaluación del Ministerio de Educación Nacional.
} 
Renovación Curricular]. En este sentido puede afirmarse que la Promoción Automática tiene una larga historia en el país, historia que es necesario rescatar, no sólo para comprender la norma que la hizo obligatoria a nivel nacional en la Escuela Básica Primaria Urbana en 1987, sino para entender, a través de ella, la asistematicidad y dispersión de la política educativa, y la ausencia de un proyecto escolar de largo plazo en el país.

A través de este sistema de promoción se busca destituir la repitencia de curso y la evaluación punitiva realizada solamente a través de los resultados de los alumnos; por otra parte, se pretenden instituir nuevas posibilidades de trabajo pedagógico y didáctico y nuevas prácticas de evaluación y promoción, lo cual se espera incida de manera directa en la superación de los bajos índices de eficiencia interna y de la calidad y la equidad de los sistemas educativos.

Este artículo tiene como interés fundamental hacer un seguimiento a la Promoción Automática a través de la política educativa colombiana y analizar su impacto en la Escuela Básica Primaria, con el fin de aportar elementos que permitan juzgar las posibilidades y límites de dicha estrategia, cuando van a completarse 5 años de su puesta en marcha.

En una primera parte y para contextualizar la reflexión, se analizará la polémica internacional alrededor de la Promoción Automática como medio para la superación del fracaso escolar; en la segunda parte se hará una revisión de las diferentes maneras como la política educativa colombiana ha abordado —en diferentes épocas - la Promoción Automática, en la tercera parte se establecerá un balance inicial sobre el impacto de esta medida en el país sus repercusiones en los índices de repitencia, en la calidad del logro educativo en las condiciones de la enseñanza [aspectos institucionales y estrategias docentes en el aula de clase]. Finaliza el artículo presentando algunas conclusiones e hipótesis de cierre.

\section{Promoción Automática y fracaso escolar: la polémica a nivel internacional}

El problema del fracaso escolar y su relación con diferentes sistemas de promoción no ha sido objeto de estudios sistemáticos ${ }^{3}$. Dentro de los límites del presente estudio hemos tenido acceso a tres documentos que pasan revista a diferentes investigaciones relacionadas con la temática; el primero titulado: "Los determinantes del rendimiento escolar: reseña de la investigación en los países del tercer mundo" de Shiefelbeim, E. y Simmons, J. (1978) estudio que hace un inventario y análisis del conjunto de investigaciones empíricas que a gran escala y dentro del enfoque de Función de Producción Educativa [FPE] $]^{4}$ han sido realizadas en África, Asia y América Latina. Dentro

\footnotetext{
${ }^{3}$ Fracaso escolar es un concepto genérico que se utilizará en este documento para significar no sólo los problemas escolares de los estudiantes [no acceso, deserción, repitencia, bajo rendimiento académico], sino también las diferentes maneras como esta sintomatología expresa la incapacidad de la escuela para responder a sus responsabilidades sociales:

- Eficiencia interna: garantizar el acceso y permanencia.

- Calidad y pertinencia de las estrategias de enseñanza y de los aprendizajes escolares.

- Equidad: distribución de las oportunidades de aprendizaje de acuerdo a las necesidades de los diferentes sectores sociales.

4 "El concepto FPE trata a la educación, generalmente a la escolar, como un proceso en el cual los "insumos" de mano de obra y capital -[tales como maestros, libros, edificios) se combinan para producir "resultados" [por ejemplo aumento en el conocimiento o, más sencillamente graduados] en la forma más eficiente". MYERS, R. en el prólogo a SHIEFELBEIM, E. Y SIMMONS, J. [1987: 6].
} 
de este trabajo se analizan variables que podrían estar determinando el rendimiento estudiantil las cuales se agrupan en tres categorías: Recursos y procesos escolares (Edificación, materiales educativos, etc.), atributos del maestro [edad, sexo, años de formación, títulos, experiencia profesional, etc.] y rasgos del estudiante (Condiciones socioeconómicas y culturales de la familia, nivel de nutrición, condiciones psíquicas, etc.). Dentro de esta revisión no aparecen referencias a los sistemas de Promoción como factor relacionado con el fracaso o el éxito escolar.

El segundo estudio es el realizado por TEDESCO, J.C. (1981) "Modelo Pedagógico y fracaso escolar" en el cual el autor, después de revisar las investigaciones realizadas en América Latina acerca de las causas generadoras del fracaso escolar, señala que no es posible determinar el predominio unidimensional de alguno de los diferentes tipos de causas. El autor analiza lo que las investigaciones latinoamericanas han planteado sobre el fracaso escolar, para lo cual divide las causas de este fenómeno en diferentes tipos según sean exógenas o endógenas al sistema educativo y según se refieran a aspectos materiales o culturales.

El documento ubica el problema de los sistemas de promoción como una de las variables endógenas organizativas. Como se señaló más arriba, se reconoce la falta de conocimiento que en América Latina existe sobre esta modalidad. ". . . Resulta claro que el problema de la Promoción Automática no está suficientemente evaluado (en América Latina) y que, en todo caso, su implantación si se mantienen constantes las demás variables de orden pedagógico, no resuelve el problema sino que, a lo sumo lo posterga" TEDESCO, J. C. (1981: 142).

El tercer trabajo también es latinoamericano; en él MUÑOZ, C. y LAVIN, S. (1988) además de considerar los estudios sobre los efectos de los factores de carácter extraescolar que intervienen en la determinación de iniciar y terminar el ciclo educativo, incorporan estudios que se ocupan de la calidad de dicho ciclo, principalmente, los que estudian los procesos de enseñanza-aprendizaje que ocurren al interior del aula. Este documento hace explícita referencia a las normas de promoción como "uno de los determinantes operativos.., que tiene directa repercusión en los índices de reprobación e indirectamente en los de deserción" MUÑOZ, C. y LAVIN, S. (1988: 139). De acuerdo con este trabajo, en América Latina "no se han detectado investigaciones que estudien específicamente la relación retención-normas de promoción, sino sólo estudios descriptivos que comparan dichas normas entre los distintos países latinoamericanos" (139).

A nivel de la definición de políticas y normas de promoción pueden observar-se en la región dos tendencias generales respecto a la conveniencia o no de implantar sistemas de Promoción Automática:

- Quienes optan por sistemas de promoción que privilegian la repetición como estrategia pedagógica que permitiría entre otras cosas: nivelar los grupos y así facilitar el trabajo docente y el adecuado desarrollo del grupo, ayudar a que los alumnos inmaduros se ubiquen en grupos ajustados a su desarrollo, de tal manera que sin presiones vayan a su propio ritmo. La promoción al grado siguiente actuaría como factor motivacional de emulación tanto para el maestro como para el estudiante.

Los críticos de estos sistemas de promoción señalan que de acuerdo con las investigaciones, la variable más fuertemente relacionada con la deserción es la reprobación. Además se afirma desde la investigación que "repetir un año escolar no parece 
ayudar al niño a dominar las habilidades académicas de ese mismo grado. Por el contrario, la ansiedad respecto al fracaso académico de un alumno, que con frecuencia se refleja en la presión de los padres, crea muchas veces problemas de desadaptación social y personal" HADDAD, W. (1991). De otra parte, la reprobación sería inconveniente puesto que además de lo ya señalado: limita la capacidad de acceso al sistema, afecta la equidad en la distribución de oportunidades de aprendizaje (afecta mucho más directamente a estudiantes de áreas rurales y urbano-marginales) e incrementa ineficientemente los costos de la educación.

- Del otro lado se ubican quienes apoyan los sistemas de promoción automática los cuales se legitiman (además de los argumentos señalados arriba para criticar a los sistemas basados en la repetición), en tesis como las siguientes: la reprobación no hace que mejore el nivel de homogeneidad dentro de los grupos; con o sin reprobación los grupos seguirán siendo heterogéneos. La repitencia además de los efectos nocivos en el autoconcepto del niño, genera un desfase entre la edad cronológica y el grado en el que se encuentra un alumno lo cual dificulta mucho mas sus posibilidades de adaptación social. El ser o no promovido no es un elemento que permita conocer el grado de aprendizaje de un alumno, las evaluaciones y los sistemas de decisión respecto a la promoción no proporcionan un indicador válido y confiable para establecer el nivel de conocimiento de un alumno.

Los argumentos en contra de la Promoción Automática (esbozados por la primera tendencia) se basan en que ésta supuestamente disminuye el rendimiento. estándar del grupo, hace decrecer la motivación tanto del maestro como del alumno, acrecienta los problemas pedagógicos en el aula al obligar al docente a manejar grupos heterogéneos, además de que podría generar otro tipo de frustración en el alumno al ubicarlo en una situación de aprendizaje y un grupo que trabaja temáticas que van más allá de su comprensión ${ }^{5}$.

De acuerdo con MUÑOZ, O. y LAVIN, S. (1988: 140) "faltan evidencias suficientemente sustentadas que permitan establecer conclusiones definitivas sobre esta polémica. Sería de importancia para la definición de políticas en la región estudiar sistemáticamente los efectos de la Promoción Automática (y sus diferentes modalidades) en la repitencia y el rendimiento en los países que la han implantado y que, o bien la han suspendido, o la mantienen: Panamá. Puerto Rico, El Salvador, Venezuela, Ecuador, Brasil, Costa Rica, Chile y ahora Colombia".

Lo que parece evidente, desde la revisión de los estudios realizados es que la solución del problema del fracaso escolar no puede reducirse a medidas administrativas relacionadas con los sistemas de promoción, como señala HADDAD, W. (1991: 149) "promover o no promover no es el problema. El verdadero problema es como mejorar el nivel de los alumnos de bajo aprovechamiento y en última instancia cómo evitar la reprobación"6. Desde el punto de vista del autor citado, las medidas preventivas implicarían un replanteamiento profundo en los procesos pedagógicos y didácticos así:

- La estructura curricular, la cual más que por años y grados escolares debería organizarse en pequeñas unidades discretas procesualmente articuladas, de tal manera que la retroalimentación se diera en períodos cortos y manejables. Lo anterior implicaría

\footnotetext{
${ }^{5}$ Ver al respecto un resumen de argumentos pro-contra de la Promoción Automática en LATAPI, P. [1991: 92].

${ }^{6}$ El subrayado es nuestro.

Digitalizado por RED ACADEMICA
} 
la superación de la organización escolar por grados homogéneos y con currículos anuales.

- Establecer políticas de tiempos diferenciales de enseñanza para los alumnos que poseen habilidades diferentes. La investigación ha mostrado que el alcanzar determinado nivel de aprovechamiento requiere de diferentes cantidades de tiempo real, dependiendo de cada alumno, y bajo diferentes condiciones de enseñanza.

- Replantear la función de la evaluación educativa de tal manera que su foco de atención y utilización no sea solamente la certificación y la promoción, sino más bien el "estimar el grado de aprendizaje de los alumnos, y así diagnosticar la efectividad de los métodos de enseñanza y lo adecuado de los materiales, con un mecanismo de retroalimentación que introduzca mejoras siempre que se requiera" HADDAD, W. (1991: 151).

Los planteamientos anteriores guardan relación con lo planteado por MUÑOZ, G y LAVIN, S. (1988: 140) quienes señalan que "la P.A. aplicada sólo como norma pero sin apoyos de nivelación pedagógica, aplicación de técnicas de individualización o apoyos psicopedagógicos dentro o fuera del aula, no constituye en sí una solución viable al problema del bajo rendimiento escolar".

En la misma línea BRAVO, L. SALAS, S. (1975: 34) afirman: "ni un sistema de P.A., ni un sistema de repeticiones reiteradas basado en el nivel de conocimientos logrados serían la solución a las dificultades. Solamente un sistema de educación flexible y diferenciado, a partir de un diagnóstico de las dificultades de aprendizaje, sería la solución adecuada que permitiría conocer los problemas que cada niño presenta y establecer un plan adecuado".

Dentro del contexto anterior, se ha considerado necesario e importante analizar la experiencia colombiana en la puesta en marcha y el desarrollo e impacto de esta política educativa en la eficiencia del sistema educativo, en la calidad del logro académico y de las condiciones de la enseñanza y, finalmente, en la capacidad de la Escuela Básica Primaria para distribuir de manera equitativa las oportunidades de aprendizaje.

\section{La Promoción Automática en la política educativa colombiana}

Durante las últimas décadas, la Escuela Básica Primaria estatal ha sido objeto importante de atención para el Ministerio de Educación Nacional. Los diferentes gobiernos han venido desarrollando diferentes programas dentro de los cuales los procesos de promoción escolar han cobrado gran importancia. En el contexto de las diferentes reformas la Promoción Automática se ha planteado como una de las alternativas generadas para superar los problemas de acceso, repitencia, rendimiento y equidad educativa ${ }^{7}$.

\footnotetext{
${ }^{7}$ Las más importantes reformas a la educación básica emprendidas y desarrolladas durante estos años han sido:

— La iniciación con carácter experimental de la Escuela Unitaria en 1961.

—El decreto 1710 de 1963 mediante el cual se unificó la educación básica primaria.

- La puesta en marcha del programa Escuela Nueva en 1975, como adaptación de la modalidad de la Escuela Unitaria.

- El programa de mejoramiento cualitativo de la educación [19761 que ha tenido como uno de sus desarrollos más importantes la Renovación Curricular.

—La promulgación y puesta en marcha de la PA en 1987. 
Sin embargo el significado de la Promoción Automática no ha sido unívoco en la política educativa colombiana, por el contrario, a lo largo de la legislación ha tenido diferentes connotaciones asociadas a los diferentes programas dentro de los que aparece y a las diferentes problemáticas que estos pretenden resolver, veamos:

\subsection{Promoción Automática elemento estratégico del modelo pedagógico de Escuela Unitaria}

Promoción Automática es un concepto que surge de manera explícita en el contexto del programa Escuela Unitaria, programa que se empieza a implementar en 1961 en diferentes países latinoamericanos para responder a los problemas de repitencia, deserción y baja calidad de la educación primaria rural en áreas de baja densidad de población. El modelo de Escuela Unitaria había sido experimentado con éxito en zonas rurales apartadas de algunos países europeos. De ahí que la UNESCO, en la primera reunión internacional de Ministros de Educación celebrada en Ginebra, Suiza en 1961, recomendara la puesta en marcha de esta modalidad escolar en los países del tercer mundo con problemas de cobertura en el área rural.

El modelo pedagógico ${ }^{8}$ de Escuela Unitaria aparece como una alternativa para resolver problemas particulares de los estudiantes de las áreas rurales apartadas. Situaciones como el reducido número de niños de muy diferentes edades, ritmos y estadios de aprendizaje que deben ser atendidos por un solo maestro; la necesidad de garantizar la permanencia de los estudiantes en la institución aun cuando las necesidades agrícolas y climáticas les exija ausentarse por períodos; la urgencia de establecer los cinco grados de la escuela básica, reclaman soluciones que el modelo de la Escuela Unitaria pretende resolver.

Por otro lado, el problema de la promoción ya se había manifestado en el área rural antes de la implementación de la Escuela Unitaria: los inadecuados manejos del proceso de promoción estaban afectando la posibilidad de acceso de muchos niños a la escuela y estaban fomentando altas tasas de repitencia y deserción. De acuerdo con el Ministerio de Educación Nacional "los maestros rurales prestan poca o ninguna atención a las promociones o las ejecutan de manera caprichosa, sin evaluar previamente el rendimiento del niño. Es un vicio de la escuela rural colombiana que se explica por las siguientes razones: el maestro no se preocupa en promover determinado número de alumnos porque no existe el grado siguiente, porque la movilidad de los alumnos le procura más trabajo en su labor, y personalmente le conviene mantener sus cursos repletos de alumnos para justificar la existencia de la escuela, y finalmente, porque es incapaz de elaborar y aplicar pruebas objetivas. Estos aspectos negativos de la escuela rural colombiana contribuyen poderosamente a la deserción escolar". MEN (1963: 19).

Frente a la situación planteada, la modalidad de Escuela Unitaria, dentro de una concepción activa de la enseñanza y con contenidos adecuados a las características de la comunidad, reformula drásticamente la estructura espacio-temporal y pedagógica de la escuela tradicional. En efecto, dentro de este modelo el aprendizaje será individualizado y

\footnotetext{
${ }^{8}$ Se habla de modelo pedagógico para hacer referencia a la manera como se articulan una concepción de escuela, enseñanza y aprendizaje, a determinadas estrategias didácticas y escolares utilizadas dentro de la institución con los estudiantes (organización del espacio, el tiempo y los contenidos escolares, uso de textos, tipo de relaciones que la escuela establece con el entorno, etc.). Ese conjunto de elementos y sus múltiples y complejas relaciones constituyen un "modelo pedagógico" cuando ellos interactúan ¿entro de un campo semántico común.
} 
facilitado por la utilización de fichas de autoinstrucción preparadas por los maestros; por otra parte, los contenidos que deben ser aprendidos se organizan en pequeñas unidades modulares, de manera secuencial y progresiva las cuales rompen con los ciclos anuales en los que se organizan los cursos y asignaturas de la Escuela Tradicional. Lo anterior implica estrategias didácticas diferentes: cada día el maestro debe preparar las guías de trabajo para cada uno de los subgrupos los que trabajarán a un mismo tiempo temáticas distintas; implica, también, sistemas novedosos de organización de los niños los cuales pueden interactuar en diferentes grupos y avanzar a diferente ritmo en cada una de las áreas de estudio [lenguaje, matemáticas, etc.]. La idea de curso dentro del cual un conjunto de niños debe avanzar a un mismo ritmo y cubrir simultáneamente todas las áreas académicas en un año escolar queda abolida.

En el contexto del modelo pedagógico de Escuela Unitaria, la Promoción Automática aparece como elemento fundamental del programa, como condición de posibilidad para desarrollar una escuela abierta y no graduada, que a la vez permite superar las dificultades que a nivel de la promoción escolar venían generándose; "en la escuela unitaria la promoción individual es automática. El alumno avanza de acuerdo con sus poderes en cada ramo y si termina el programa en la sección A, por ejemplo, pasa a la B, sin esperar las fechas de exámenes. Los niños que llegan atrasados en edad a la Escuela Unitaria, tienen la oportunidad de avanzar más rápidamente y recuperar en parte, el tiempo perdido". (MEN, 1968: 22).

En la Escuela Unitaria la idea de repetir no existe; el estudiante no tiene que volver sobre temas que ya cursó, aunque es posible que se demore un poco más que sus compañeros en una unidad; en este sentido "no entiende en efecto la Escuela Unitaria quien habla de grados en su acepción ordinaria de años escolares cerrados y piensa en ellos al organizar su trabajo". (Hernández, J. citado por BARROS, J. 1975: 11). La modalidad "no concibe promociones a fecha fija ni la repetición sistemática de un curso por un alumno, como en la escuela graduada".

(MEN, 1963: 19).

De acuerdo con lo anterior, la Promoción Automática en la Escuela Unitaria es un elemento necesario y coherente con un programa que replantea el conjunto de las prácticas de enseñanza la organización y administración escolar, las estrategias didácticas de los docentes, las actividades de los alumnos, el concepto de repitencia y fracaso. Promoción Automática no es allí un elemento aislado y puntual, por el contrario, hace parte central del modelo el cual no podría entenderse y operar sin este sistema de promoción.

\subsection{Promoción flexible en el programa Escuela Nueva operacionalización de la Promoción Automática de la Escuela Unitaria}

Aparentemente el modelo pedagógico de Escuela Nueva sigue los lineamientos centrales de la Escuela Unitaria, sin embargo, dentro de la perspectiva de ampliación de la cobertura de este modelo hacia nuevos sectores rurales apartados, se formula la necesidad de resolver algunos problemas prácticos surgidos de su experimentación. De una parte, la elaboración de las guías, en todas las áreas y para todos los grados por parte de los maestros estaba teniendo serios tropiezos por la enorme carga de trabajo que implicaba, de otra, los sistemas de promoción estaban tomando un rumbo bastante subjetivo y azaroso. Finalmente, los procesos de c. incitación y administración del programa no eran suficientemente claros. A partir de esta situación se formula la necesidad de diseñar al detalle e instrumentalizar el modelo anterior en su conjunto. 
Aunque en la teoría se afirma que se mantiene el enfoque del modelo anterior ${ }^{9}$ la organización curricular por unidades discretas sistemáticamente agrupadas en niveles y la producción central de guías comunes para todas las escuelas introducirá una muy importante modificación en el rol del maestro quien de orientador y director de la clase se convertirá en el administrador y adaptador de las guías.

Las guías se convertirán así en uno de los elementos estratégicos para el desarrollo de la acción docente. A través de ellas se garantiza un trabajo sistemático de los niños en pequeños grupos y el cumplimiento de los objetivos planteados por ellas. En las guías se expresa una estructura y organización curricular para cada una de las áreas del conocimiento por niveles, los que a su vez se subdividen en fascículos (conjunto de materiales para abarcar cada nivel) y éstos en unidades las cuales contienen objetivos que se desarrollan secuencialmente a través de un conjunto de actividades.

La promoción se realiza por unidades, una vez que se ha evaluado y calificado el cumplimiento de cada una de éstas por cada uno de los alumnos. A través de la guía como herramienta, el alumno que a causa de factores climáticos, de cosecha o algún otro motivo debe abandonar la escuela por un período no pierde el curso; al regresar continua a partir del punto en que se encontraba. Dentro del contexto de este programa, al igual que en la Escuela Unitaria, se rompe la estructura de grado, de año escolar, de pérdida y repetición del curso, del avance homogéneo de todos los alumnos en todas las áreas. La evaluación, la calificación y la promoción pueden darse en cualquier momento del año. El alumno puede interactuar con compañeros diferentes en las distintas áreas de acuerdo con el avance que haya logrado en cada una de ellas.

\subsection{La Promoción Automática en el contexto de la Renovación Curricular}

Para la década del 70 la expansión de la cobertura educativa había alcanzado importantes avances en el país. Sin embargo este proceso había traído consigo un importante deterioro en la calidad de la educación. La preocupación del Ministerio de Educación Nacional ya no tiene como eje la ampliación de la cobertura, sino más bien la cualificación de la educación. En 1976 y con el fin de mejorar cuantitativa y cualitativamente la educación sistematizando los procesos educativos, mediante el empleo y generación de tecnología educativa, para ampliar las condiciones de acceso a la educación en forma equitativa se presentó al país el "Programa Nacional de Mejoramiento Cualitativo de la Educación". Uno de los elementos centrales del programa lo constituyó la Renovación Curricular la cual se proponía garantizar la prestación del servicio en el sector rural y marginal y retener al alumno en el sistema, mejorando su rendimiento.

La Renovación Curricular, de acuerdo con su concepción ${ }^{10}$, pasó por un proceso de experimentación que se inició en 1978 y terminó con el decreto de generalización en 1984, durante estos seis años se desató en el país una fuerte polémica alrededor de los fundamentos e implicaciones de la racionalidad y el enfoque educativo que sustentaba la reforma. La Renovación Curricular ha sufrido importantes cambios a lo largo del proceso de experimentación y generalización, no solamente en los contenidos de algunos de los

\footnotetext{
9 "Los objetivos y los principios de Escuela Nueva son los mismos de la Escuela Unitaria; la diferencia radica en los mecanismos de Ejecución del programa Escuela Nueva, a través de los cuales se busca que el maestro rural ofrezca y maneje los cinco grados de la escuela, que el niño realmente avance a su particular ritmo de aprendizaje [promoción flexible] y sea el protagonista del proceso, y que la comunidad participe en forma dinámica de las actividades de la escuela". [COLBERT, V., MOGOLLON O. 1987: 7].

10 "La RC es un proceso permanente y abierto a la continua adaptación y ajuste. No se presenta como un producto terminado. Es un proceso que obedece a la concepción de apertura y flexibilidad del currículo". [MEN 1986: 141.
} 
planes de estudio sino también en sus fundamentos generales. De ahí que estudiar la manera como se planteó el sistema de promoción a su interior, implica reconocer estas modificaciones.

La Renovación Curricular comparte con la Escuela Unitaria y la Escuela Nueva algunos de sus principios pedagógicos básicos, por ejemplo: adecuación de los programas al medio, enseñanza integrada de las disciplinas, relaciones estrechas escuela-comunidad, métodos activos, cambios en las concepciones y prácticas evaluativas. Estos principios sin embargo, han sido asumidos de manera diferente y han tenido grados de aplicación muy distintos.

A pesar de que en algunas normas de la Renovación Curricular se plantea explícitamente la Promoción Automática y la Promoción Flexible para algunos grados de la escuela básica primaria, el tratamiento dado a los dos conceptos de promoción es, en este contexto, muy diferente al desarrollo en Escuela Unitaria y Escuela Nueva.

- La primera diferencia fundamental se da en la formulación de la Promoción Automática. Mientras en Escuela Unitaria y Escuela Nueva la Promoción es un elemento derivado y coherente con toda una estructura pedagógica y didáctica compleja fuera de la cual no podría entenderse, dentro del Programa de Mejoramiento Cualitativo de la Educación la Promoción Automática se plantea como un problema administrativo. La norma que la define ${ }^{11}$ aparece en un decreto en el cual no se plantea un programa escolar sino una reforma administrativa.

- Posteriormente (1982) mediante la Resolución 20469 se autorizó la experimentación curricular para el nivel de educación básica en el Distrito Especial de Bogotá en los grados primero, segundo y tercero. En dicha resolución se define que la promoción en estos grados será automática y a pesar de que en esta norma la promoción aparece ligada a algunos cambios pedagógicos 1horas de clase de 60 minutos], nuevos programas, nuevo enfoque evaluativo y, sin embargo, la diferencia del concepto Promoción Automática con la formulación de Escuela Unitaria y Escuela Nueva es evidente. Dentro de la Renovación Curricular se mantiene la estructura de grados, de año escolar, de cursos homogéneos y, lo más importante, la noción de repitencia y pérdida de año escolar.

-De acuerdo con algunos documentos del MEN y en consultas realizadas a nivel nacional se encontró "un enfático rechazo a que haya Promoción Automática de primero a tercer grado sin aclarar el significado del término y sin una previa investigación al respecto que estudie las condiciones necesarias para garantizar la calidad del aprendizaje" ${ }^{12}$. Tal parece que esta tendencia jugó un papel importante en la definición de Flexible que se le dará a la Promoción de primero a tercer grado en la norma sobre generalización de la Renovación Curricular (Res. 17486 de 1984) ${ }^{13}$.

\footnotetext{
11 “La promoción en la escuela básica primaria será automática y será reglamentada posteriormente" [Decreto 088 de 1976 Art. 8]. Este es un decreto por medio del cual se organiza administrativamente el Ministerio de Educación Nacional.

12 MEN [1983]. "Informe del tercer seminario de coordinadores de los CEP a nivel nacional, Bogotá, diciembre de 1983". Mimeo.

13 "Art. 4. La evaluación de los tres primeros grados de la básica primaria deberá prestar especial atención a la edad y al ambiente social y cultural del que proceden los alumnos a fin de garantizarles avanzar a su propio ritmo de aprendizaje, reduciendo así las tasas de repitencia y deserción frecuentes en estos grados de la educación básica primaria. A este fin y para asegurar la Promoción Flexible de los estudiantes no regirán para estos 3 grados los períodos de evaluación ni los valores ponderados de las calificaciones asignadas a los demás grados y niveles.
}

Digitalizado por RED ACADEMICA 
A pesar de que la Renovación Curricular estableció que dentro del programa "La evaluación adquiere un sentido positivo y estimulante, en cuanto su función es detectar fallas y aciertos y producir información para introducir los correctivos necesarios que garanticen el progreso del estudiante". . . .No se trata de eliminar al estudiante dentro de un sistema competitivo y selectivo que descarta a los menos aptos, sino de evitar que fracase" (Ministerio de Educación Nacional. 1983). Queda claro sin embargo, que al mantener los grados escolares, el año escolar, la fecha única de promoción al final del curso y la noción de pérdida de año y repitencia, se mantienen intactas las estructuras básicas de la escuela tradicional. Por lo tanto, el concepto de Promoción Flexible tiene en esta norma una connotación completamente diferente a la señalada por Escuela Nueva. En este sentido puede afirmarse que las propuestas de promoción definidas por la Renovación Curricular -aunque retoman los nombres - se apartan de los planteamientos básicos que estos conceptos habían alcanzado en las políticas educativas definidas para el área rural.

\subsection{La Promoción Automática (Decreto 1469-1987) norma obligatoria para la Escuela Básica Primaria urbana}

La Promoción Automática con norma Nacional aparece en Colombia dentro de un contexto muy particular; en primer lugar, de auge del Movimiento Pedagógico Nacional ${ }^{14}$ que como parte de la crítica hacia la Renovación Curricular centraba su atención en la autonomía intelectual y profesional del maestro. En segundo lugar, en la investigación educativa colombiana había adquirido un estatuto importante la perspectiva pedagógica, el interés por el estudio de las relaciones maestro-alumno en el aula de clase y la institución, y la utilización de métodos cualitativo-comprensivos. En tercer lugar, dentro del Ministerio de Educación Nacional se estaba en un proceso de apertura hacia la crítica presentada a la Renovación Curricular. Además estaba en marcha la discusión sobre la descentralización educativa y existía la propuesta de un "Plan Nacional de evaluación" 15 para la transformación de las concepciones y prácticas evaluativas.

En este marco, en el año 1987 el gobierno plantea el programa "Acción Nacional educativo-cultural" ${ }^{16}$. Una de las preocupaciones de este programa se relaciona con el acceso y la retención de la población dentro del sistema escolar, el programa considera que existe una relación muy estrecha entre los fenómenos de deserción y repitencia y el modelo escolar tradicional, el cual debe ser replanteado.

\footnotetext{
Parágrafo 1. Para que los alumnos de cada uno de estos grados pueda ser promovido al grado siguiente se requiere que logren los objetivos mínimos de aprendizaje en las áreas de Español y Matemáticas. Con la pérdida de estas áreas el alumno no podrá ser promovido al grado siguiente. En cambio habrá mayor flexibilidad para el alumno que no logre los objetivos en las demás áreas, pero que a juicio del maestro y los directivos del centro docente los pueda alcanzar en el grado siguiente, en tal caso podrá ser promovido a dicho grado".

${ }^{14}$ El Movimiento Pedagógico surge de experiencias innovadoras de maestros de base, recibe el apoyo y oficialización por parte del sindicato de maestros FECODE y es definido como "una corriente de ideas y de intentos prácticos. . . que expresan nuestra voluntad de retomar la palabra, con el fin de recuperar el dominio teórico y práctico sobre nuestro quehacer". Ver al respecto "Fundamentos y propósitos del Movimiento Pedagógico". Revista Educación y Cultura No. 1, julio, 1984.

${ }^{15}$ El Plan Nacional de Evaluación es un programa de trabajo propuesto por un equipo interinstitucional Ministerio de Educación Nacional, Universidad Pedagógica Nacional y DIECEP, equipo que a partir de un diagnóstico acerca de la evaluación escolar en Colombia presentó esta propuesta orientada a la transformación de las concepciones y prácticas evaluativas en la escuela básica y media del país.

${ }^{16}$ Ver al respecto Yepes Parra, A. (1988). "La escuela como proyecto cultural". El Campesino, Bogotá, Ministerio de Educación Nacional.
} 
La norma de Promoción Automática ubicada en esa perspectiva centra su atención en la evaluación escolar partiendo del supuesto que transformaciones en las prácticas de evaluación conllevan cambios en el aprendizaje, en la enseñanza, en los criterios de promoción, en el rol del docente y en la organización escolar ${ }^{17}$. De acuerdo a lo planteado por el Equipo Interinstitucional de Evaluación que se constituyó en el soporte técnico de la medida "Si se aprenden datos es porque se evalúan datos, por tanto si logramos evaluar otras cosas y de otras formas también lograremos otros aprendizajes" (1987: 83).

La Promoción Automática señala la necesidad de replantear la concepción de evaluación y ampliar los objetos de evaluación retomando algunos de los planteamientos de la Renovación Curricular. Se pretende superar la concepción de evaluación entendida como calificar, promover y certificar al niño para ubicar la mirada evaluativa en la totalidad de los procesos escolares, superando el tradicional énfasis que tiene como eje los resultados de los niños en las diferentes áreas.

Según la propuesta de la Promoción Automática los problemas de aprendizaje no dependen exclusivamente de las condiciones de los alumnos, están íntimamente relacionados con estrategias didácticas de los docentes en el aula y con las condiciones materiales y culturales de la escuela en cuanto institución. De acuerdo a lo anterior la evaluación deberá atender estos nuevos elementos: en el niño deberán evaluarse sus procesos de desarrollo más que los logros en las diferentes áreas, además se evaluarán los procesos pedagógicos (pertinencia y efecto de las estrategias de enseñanza de los docentes en los aprendizajes de los niños) y, finalmente los procesos organizacionales y administrativos para determinar el efecto del "clima escolar" en los logros de los estudiantes.

Esta nueva concepción de evaluación que enfatiza procesos, señala la necesidad de un cuestionamiento y transformación permanente de las prácticas docentes en el aula de clase y de los sistemas de organización escolar para que se adecuen permanentemente a las condiciones de los niños y su ambiente. De otra parte propone actividades de recuperación como recurso pedagógico que permita compensar en el transcurso del año las dificultades de los alumnos que presentan algún déficit en su proceso de desarrollo. También en relación con el énfasis otorgado a los procesos se abolió la calificación numérica y fue reemplazada por una escala conceptual: Excelente, Bueno, Aceptable e Insuficiente ${ }^{18}$.

La norma introdujo, además de lo anterior, dos importantes innovaciones: el cambio de la tradicional libreta de calificaciones numéricas por un "Boletín descriptivo-explicativo" en el que se debe comunicar a los padres de familia de manera cualitativa los avances y dificultades del niño en los procesos escolares. También señaló la necesidad de crear en cada institución de Básica Primaria "comités de evaluación" encargados de la "orientación y control de los procesos evaluativos dentro del establecimiento" (Res. 13376-Octubre de 1988, Art. 4).

\footnotetext{
17 "La evaluación constituye un elemento nodal en la trama escolar, elemento que expresa y establece las relaciones de poder en la escuela y define, por ende, lo que en ella se hace cotidianamente frente al saber y la vida social". EQUIPO MEN-UPN [1988]. "Evaluación y cambio escolar" Boletín informativo del plan nacional de evaluación. Bogotá, No. 1, junio de 1988.

${ }^{18}$ Debe subrayarse que en el programa Escuela Nueva y su promoción flexible también se propone una escala de calificaciones, pero a diferencia de la norma nacional de Promoción Automática no existe el "insuficiente" dentro de ese contexto no se concibe que el niño "pierda".
} 
Debe resaltarse que la norma no es automática en sentido estricto, pues señala una serie de condicionantes para llevar a cabo la promoción: el cumplimiento de los objetivos mínimos en cada una de las áreas, la asistencia al por lo menos $80 \%$ de la actividad escolar y la consideración acerca de las capacidades de los estudiantes ${ }^{19}$.

Como puede observarse, la Promoción Automática como norma Nacional es fundamentalmente una norma de evaluación. A diferencia de lo que significó la promoción en la Escuela Unitaria o la Escuela Nueva en las que la propuesta de evaluación y promoción surgían del modelo educativo, en esta propuesta se espera que el modelo pedagógico emerja de un nuevo tipo de evaluación. Por esta razón no se presenta articulada a un modelo pedagógico integral en el que se inscriba el replanteamiento evaluativo y escolar que se propone. La norma sobrevaloró la capacidad del factor evaluativo como supuesto generador de transformaciones globales en la escuela.

En la política de Promoción Automática se expresa la falta de continuidad y coherencia en las políticas educativas dirigidas a la escuela primaria colombiana. No sólo se presenta un manejo polisémico, ambiguo y desorientador para los docentes acerca de los tipos de promoción escolar que se deben implementar, sino que evidencia una política educativa que se mueve entre modelos educativos diferentes; mientras la Renovación Curricular enfatizaba la enseñanza en cada una de las áreas como la tarea fundamental de la escuela y la didáctica como estrategia, la Promoción Automática, enmarcada en la política "Acción Nacional educativo-cultural", enfatiza la función socializadora de la escuela y su papel institucional como formadora de valores cívicos y democráticos, no sólo en los niños que asisten a la escuela, sino también en la comunidad circundante.

Cada uno de estos énfasis propone diferentes demandas a la escuela, sin generar articulaciones entre los diferentes requerimientos y sin plantearse seriamente las relaciones entre la formación social y cívica con la enseñanza de saberes especializados en la especificidad del espacio escolar.

Llama la atención la manera ahistórica como ha procedido la política educativa en el caso de la Promoción Automática el manejo equívoco del concepto, la falta de continuidad y sistematicidad en los planes y programas que pretenden afectar a la Escuela Básica Primaria. Una medida que busca transformaciones globales de la escuela pero que no retoma esfuerzos y experiencias anteriores, dejando de lado la posibilidad de articularse a ellas (Escuela Unitaria, Escuela Nueva), que no parte de un diagnóstico de la escuela realmente existente (con sus enormes diferencias de acuerdo al contexto socioeconómico y cultural en el que se ubica), es una medida cuyos límites en el impacto de la dinámica escolar son previsibles.

\section{Algunos efectos de la Promoción Automática (Decreto 1469 de 1987) en la Escuela Básica Primaria colombiana}

La Promoción Automática (Decreto 1469 de 1987) es una política que ha generado un buen número de investigaciones, la mayoría de ellas se han realizado por iniciativa gubernamental y han encontrado apoyo financiero estatal para su realización.

\footnotetext{
19 Art. 2. literal b "Promoción Automática: El proceso por medio del cual después de un seguimiento permanente de evaluación escolar, todo niño que cursa un grado en el nivel de básica primaria, es promovido al grado siguiente al finalizar el año lectivo o antes, si sus capacidades y logros se lo permiten". Art. 7. "En caso de que el alumno acumule el $20 \%$ o más de las fallas no justificadas con respecto al número de horas-clase realizadas, no se considerará cursado el grado respectivo".
} 
Se han realizado dos tipos de estudios: unos para evaluar su puesta en marcha y otro para estudiar su impacto a nivel de eficiencia y calidad de la Escuela Básica Primaria, Por otra parte, los resultados del Sistema Nacional de Evaluación de la Calidad Escolar que se están publicando por esta fecha, —aunque no tenían como objetivo evaluar la medida, se constituyen en importantes indicadores de lo que ésta ha generado a nivel del logro académico ${ }^{20}$.

No es interés de esta parte del documento presentar un estado del arte sobre el tema, sino más bien realizar una lectura transversal puntual y sintética de los resultados de estos estudios, de tal manera que puedan señalarse, a partir de ellos, algunos de los efectos de la medida y algunas hipótesis explicativas respecto a la situación del "fracaso escolar" en la Escuela Básica Primaria colombiana y acerca del papel jugado por la norma de Promoción Automática esta situación.

\subsection{Promoción Automática y tasas de repitencia escolar}

No existe en el momento información actualizada, clara y precisa respecto a la magnitud de la repitencia escolar en el país. A partir de la información existente ${ }^{21}$ se presentarán algunos de los datos cuantitativos que se conocen en la perspectiva de tener una idea menos unilateral sobre el impacto de la Promoción Automática en la eficiencia del sistema. Se compararán los datos anteriores a la puesta en marcha de la norma con los posteriores a la vigencia de la misma con el fin de poder tener una idea de los efectos de la medida.

Los datos existentes en el DANE —correspondientes al período 1981-1987— señalan una tendencia histórica a la baja en las tasas de repitencia en Colombia durante la década del 80 y antes de la puesta en marcha de la Promoción Automática.

\footnotetext{
${ }^{20}$ Son estas investigaciones: BARCO, M.T.Cls [1991], BATISTA E. Cls. L1989], BONILLA, N. [1991], CABALLERO, P. [1989], FORERO, J. [1991], OROBIO, H. [1990], ORTIZ, N. [1994, PINILLA, G., VILLEGAS, L.A. [1991], POSADA, R., PAVA, C. [1994. Debe subrayarse que 8 de estas 13 investigaciones corresponden a un proceso realizado de manera coordinada y simultánea por equipos de investigación de ocho facultades de Educación de siete diferentes regiones del país que respondieron a una convocatoria Nacional realizada por el Ministerio de Educación Nacional y Colciencias con el apoyo del Departamento Nacional de Planeación. El proceso contó con la asesoría de un equipo interinstitucional Ministerio de Educación Nacional-Centro de Investigaciones de la Universidad Pedagógica Nacional. Ver al respecto ROJAS, M., CAMARGO, M. VILLAR, R. "Informe final del equipo coordinador del concurso de méritos para el seguimiento a la puesta en marcha de la Promoción Automática". Bogotá, 1991, mimeo.

- Por otra parte, entre octubre de 1991 y junio de 1992 se llevó a cabo un estudio nacional sobre repitencia escolar en la escuela estatal colombiana. Dicho estudio tenía como uno de sus objetivos "Indagar acerca del impacto de la Promoción Automática en las prácticas pedagógicas de los docentes y la repitencia escolar". (MCGUINN, N. Cls. 1992: 3). Este estudio trabajó con una muestra intencional de 180 escuelas en 56 municipios y 19 departamentos.

${ }^{21}$ En el momento existen tres tipos de datos: los recogidos por el DANE, como se ha dicho, con información hasta 1987 (DANE 1991), los resultantes de las proyecciones realizadas a través de modelos estadísticos por [Shiefelbein, E. y Heikkinen, 5. 1991] y los recogidos en el trabajo de campo sobre "La repitencia escolar en Colombia". (MCGUINN, Cls. - 1992).
} 
Tabla 1

Tasas de repitencia escolar 1981 - 1987 (DANE 1991)

\begin{tabular}{|c|c|c|c|c|c|}
\hline AÑO & GRADO 1 & GRADO 2 & GRADO 3 & GRADO 4 & GRADO 5 \\
\hline $\mathbf{1 9 8 1}$ & $22.1 \%$ & $15.5 \%$ & $12.1 \%$ & $10.0 \%$ & $6.8 \%$ \\
\hline $\mathbf{1 9 8 4}$ & $19.3 \%$ & $13.4 \%$ & $10.7 \%$ & $9.0 \%$ & $5.8 \%$ \\
\hline $\mathbf{1 9 8 7}$ & $17.7 \%$ & $10.7 \%$ & $9.4 \%$ & $9.2 \%$ & $5.7 \%$ \\
\hline
\end{tabular}

De otra parte, el estudio sobre repitencia (MCGUINN, Cls - 1992: 13) en las entrevistas a directivos, indagó acerca de las estadísticas sobre repitencia y deserción para los años 1987-1991. Los datos que presentaremos corresponden a solo 98 establecimientos de los 180 de la muestra (54.4\%), que fueron capaces de proporcionar información para la mayor parte de los años.

Tabla 2

Tasas de repitencia escolar 1987 - 1991 de acuerdo a los reportes De los directores de escuela

\begin{tabular}{|c|c|c|c|c|c|}
\hline AÑO & GRADO 1 & GRADO 2 & GRADO 3 & GRADO 4 & GRADO 5 \\
\hline $\mathbf{1 9 8 7}$ & $19.04 \%$ & $11.2 \%$ & $9.79 \%$ & $8.29 \%$ & $4.5 \%$ \\
\hline $\mathbf{1 9 8 8}$ & $14.15 \%$ & $7.4 \%$ & $6.37 \%$ & $5.76 \%$ & $1.8 \%$ \\
\hline $\mathbf{1 9 8 9}$ & $15.71 \%$ & $8.4 \%$ & $7.42 \%$ & $5.13 \%$ & $3.5 \%$ \\
\hline $\mathbf{1 9 9 0}$ & $17.54 \%$ & $8.5 \%$ & $7.99 \%$ & $6.75 \%$ & $3.2 \%$ \\
\hline $\mathbf{1 9 9 1}$ & $17.85 \%$ & $9.7 \%$ & $7.94 \%$ & $4.66 \%$ & $2.5 \%$ \\
\hline
\end{tabular}

De acuerdo con esta información la Promoción Automática que entró en vigencia en el año de 1988 produjo una baja importante en las tasas de repitencia para ese año y el siguiente, pero luego (1990-1991) pareciera que las tasas tienden a volver a la tendencia histórica señalada por la información del DANE.

Los datos anteriores (DANE 1991) - MCGUINN cls. (1992) guardan relación entre sí y se encuentran bastante distanciados de las proyecciones estadísticas realizadas por Shiefelbein, E. y Heikkinen, 5. (1991) según las cuales la magnitud del problema de la repitencía en Colombia sería crítica pues estaría cerca del $45 \%$ para el primer grado. Las estadísticas proporcionadas por los directores de escuela corroboran la tendencia histórica reportada por el DANE, el leve incremento que se presenta a partir del 90 podría explicarse como la vuelta a la tendencia después de la anómala baja de 1988 generada como efecto inmediato de la norma de Promoción Automática. (Ver tabla 2).

De otra parte, en el estudio sobre repitencia escolar MCGUINN CLS. (1992), se realizó un conteo directo de alumnos repitentes en 1991 en las escuelas de la muestra (en primero y tercero); dicho conteo muestra una tasa un tanto superior a la reportada por los directores para 1991: $23.3 \%$ para el primer grado y $10.1 \%$ para el tercero.

De acuerdo con la información anterior, no existen evidencias de que la Promoción Automática haya impactado las tasas de repitencia más allá del efecto inmediato producido en 1988 con su influjo hasta 1989. A este nivel podría afirmarse que la norma no logró su objetivo de hacer más eficiente al sistema. 


\subsection{La Promoción Automática no parece haber aportado al mejoramiento de la calidad de los aprendizajes escolares}

La calidad de los aprendizajes ofrecidos por la escuela básica primaria colombiana ha venido cuestionándose desde hace algunos años ${ }^{22}$. En el estudio sobre repitencia, citado más arriba, respecto a los logros académicos de los alumnos, se señala: "Si bien los alumnos promovidos obtienen resultados superiores a los repitentes de manera significativa, el promedio de aquellos no pasa del $50 \%$ deseado. La repitencia debe disminuir pero no se debe olvidar que un bajo índice de repitencia puede esconder la baja calidad un sistema escolar". (MCGUINN Cls. 1992).

El Sistema Nacional de Evaluación de la Calidad Escolar, por su parte, en su primer informe de resultados afirma:

"En términos generales, los resultados muestran que los niveles de calidad académica de la educación en Colombia no son satisfactorios, tanto en matemáticas como en lenguaje, en las respuestas de los estudiantes se observa una marcada tendencia a la resolución de problemas que requieren operaciones de orden inferior. Este tipo de problemas demanda altos requerimientos de memoria, pero bajas exigencias en la comprensión"

"Esto es, a pesar de que los estudiantes pueden responder a problemas mecánicos de información sobre las áreas, no pueden responder a problemas 'de aplicación' de conocimientos. Ministerio de Educación Nacional (1992: 59).

Es importante resaltar que las deficiencias encontradas a través de las pruebas de español y matemáticas son mucho más fuertes en las escuelas estatales que en las privadas, en las rurales que en las urbanas y en las regiones con más elevados índices de pobreza que en estratos socioeconómicos medios y altos (Ministerio de Educación Nacional: 1992).

Al comparar los datos de repitencia con los de calidad de los logros académicos queda en evidencia que la tendencia a la baja en las tasas de repitencia en Colombia no está asociada a un mejoramiento simultáneo en la calidad de los aprendizajes. Quizás está aconteciendo lo contrario; la baja en la repitencia ha llevado aparejada una baja semejante en la calidad. Vale la pena preguntarse cuáles son los factores que están incidiendo en los docentes para que promuevan a los estudiantes sin que éstos alcancen los aprendizajes mínimos esperados. Es conveniente señalar algunas hipótesis al respecto:

¿Acaso las nuevas tendencias -modas- pedagógicas que subrayan la necesidad de tener en cuenta las condiciones particulares de los estudiantes, sus ritmos específicos de aprendizaje, han sido interpretados como la necesidad de que bajen las expectativas y los niveles de exigencia sobre los niños de los sectores marginales y rurales?, OROBIO, $\mathrm{H}$., y Cls (1990) y ORTIZ, N. y Cls (1991) señalan que en las escuelas estudiadas por ellos escuelas estatales de sectores populares_ - "ha bajado el afán de terminar programas y la coacción y presión con la nota".

¿Hasta qué punto la Promoción Automática ha sobredimensionado la socialización como función de la escuela (independientemente de los aprendizajes escolares) y ha sido

\footnotetext{
${ }^{22}$ Ver al respecto estudios como: RODRIGUEZ, José [1982] "El logro en matemáticas y lenguaje en la educación primaria colombiana” Instituto SER de investigaciones, Mimeo, Bogotá.

Digitalizado por RED ACADEMICA
} 
leída por algunos sectores del magisterio como "lo importante es que el niño la pase bien no importa que aprenda a leer, escribir, manejar las matemáticas"?

Quizás las normas de promoción (Flexible y Automática) han generado la idea de que la reprobación no tiene más que aspectos negativos y que por lo tanto todos los alumnos deben ser promovidos sin importar qué tanto han aprendido.

\subsection{No parece que la Promoción Automática haya logrado transformar las estrategias de enseñanza}

La Promoción Automática pretendió afectar la institución escolar en su conjunto, pero especialmente las estrategias de enseñanza y las prácticas de evaluación y promoción. ¿Qué tanto ha logrado este objetivo? Se revisará lo que la investigación dice al respecto $^{23}$ :

- Los agrupamientos A pesar de lo planteado en las normas de Renovación Curricular y Promoción Automática sobre el respecto a las características individuales de los estudiantes el maestro parece estar permanentemente orientado hacia la homogenización de los ritmos de aprendizaje de los alumnos. En general, cuando se agrupa a los alumnos se hace en la búsqueda del logro simultáneo de los objetivos. No se desarrollan grupos para dar respuesta a características individuales del aprendizaje ${ }^{24}$.

- Las prácticas de evaluación del aprendizaje de lenguaje y de las matemáticas están centradas en los aspectos externos de la lectura en voz alta y de la escritura (casi siempre al dictado) para el caso de español (PINILLA, G.; VILLEGAS, L.A. 1991) y en el desarrollo de mecanicismos simples de suma, resta, multiplicación o división para las matemáticas.

A pesar de lo señalado en la norma de Promoción Automática la evaluación sigue siendo un procedimiento la mayoría de las veces terminal cuya intención es -cuando no disciplinar- en el mejor de los casos, constatar los logros académicos del programa, pero no es su interés entender los procesos de apropiación del lenguaje escrito o del pensamiento matemático e incidir en la acción pedagógica posterior para adecuarla a dichos procesos.

Se encuentra escasa preparación del maestro para detectar dificultades en el aprendizaje de los estudiantes que requieren actividades de recuperación, el maestro tampoco está formado para adecuar la enseñanza a cada problemática específica, por estas razones las actividades de recuperación terminan haciéndose de manera artesanal, casual y empírica, en muchos casos se realizan para "llenar el siguen en ellas las estrategias tradicionales convirtiéndose en "dar más de lo mismo" (BONILLA, N. Cls.; 1991). Algunas veces las actividades de recuperación funcionan como castigo tanto para padres como para niños pues asistir a ellas implica sacrificar tiempo de recreo o intensificar el trabajo en relación con los otros niños (OROBIO, H, y Cls 1990).

- Las concepciones de repitencia escolar en general, los maestros consideran que la repitencia es beneficiosa para los niños ya que a través de ella esperan que los estudiantes logren los aprendizajes necesarios o la responsabilidad ante el estudio. Ante

\footnotetext{
${ }^{23}$ En este aspecto seguiremos de cerca los resultados del análisis de la información cualitativa del estudio sobre repitencia escolar. Ver al respecto: Equipo MEN-CIUP (1992). "El mito de la escuela moderna y modernizadora" Fotocopia.

${ }^{24}$ Debe tenerse en cuenta que el maestro estatal trabaja por lo general con grupos grandes y heterogéneos, quizás la homogenización es una forma que él ha construido para facilitar su labor.

Digitalizado por RED ACADEMICA
} 
el diagnóstico que hacen los maestros de niños con limitaciones socio-culturales o afectivas, la alternativa que se les ofrece para superar estas dificultades es repetir. Repetir el grado, repetir nuevamente la información para que se "grave" en los niños, re alizar "actividades de recuperación" con las mismas estrategias de enseñanza de las actividades pedagógicas normales. Dar más de lo mismo para que a fuerza de la repetición se fije la información que la escuela pretende grabar en sus estudiantes. FORERO, J. Cls (1991).

De otra parte, la repitencia es atribuida a factores externos al hecho pedagógico: el niño, los padres, el Estado. Las estrategias de enseñanza no se ven cuestionadas. Cuando un niño no alcanza los objetivos esperados, el maestro encuentra la explicación de esta frustración casi siempre en el niño y sus problemas familiares o psicológicos; en muchas ocasiones en su falta de voluntad, atribución que es reforzada en la mayoría de los casos por la familia. (Equipo MEN-CIUP 1992).

El boletín descriptivo-explicativo traduce una preocupación por el aspecto formal de la evaluación dado que el maestro, con la Promoción Automática se interesó más por ofrecer diferentes diseños de formatos de boletín que por trabajar, comprender y apropiar alternativas evaluativas sobre el niño. Efectivamente, el maestro cambió su boletín; pero ese cambio, en muchos de los casos, se convirtió — con la ayuda del computador - en un listado de adjetivos y conceptos psicológicos más calificativo-rotuladores que descriptivo- explicativos. (OROBIO, H., y Cls 1990) y (PINILLA, G.; VILLEGAS, L. A. 1991).

\subsection{Promoción Automática y equidad}

Los diferentes estudios sobre Promoción Automática corroboran lo que otros estudios han venido señalando: las escuelas situadas en los sectores más pobres son las que presentan las peores condiciones materiales y académicas; por lo tanto, son aquellas en las que los niños tienen las menores oportunidades de aprendizaje escolar. En efecto, de acuerdo con el Departamento Nacional de Planeación, las oportunidades de aprendizaje escolar han sido mucho menores para quienes más las requerían, de tal manera que el desarrollo educativo se ha dado de manera desigual por regiones y zonas geográficas; "los perdedores en este proceso fueron las zonas rurales, los municipios de las dos costas y los grupos humanos de las zonas marginales de las cuatro ciudades más grandes". [D.N.P. - MEN 1991: 6]. Tal parece que la Promoción Automática no ha podido incidir en esta problemática. Pero la inequidad tiene que ver también con el tratamiento homogéneamente rígido en calendarios, currículos, textos estrategias de enseñanza y criterios de evaluación a los que son sometidos niños de tan diferentes condiciones socioeconómicas, culturales y personales. (Equipo MEN-CIUP 1992). No se observa en los resultados de los estudios que la Promoción Automática - que pretendía promover un trato diferenciado a los niños- haya tocado estas estructuras tan sólidas.

\section{Promoción Automática: ¿otra evidencia del fracaso del modelo escolarizador para los sectores populares?}

La norma de Promoción Automática se propuso transformar la escuela a partir del replanteamiento de las concepciones y prácticas evaluativas. A partir de allí, se aspiraba hacer de esta institución un "proyecto cultural de la comunidad", instrumento de modernización, de democratización y de desarrollo local, a través de la formación de una racionalidad técnica y crítica. De ahí la importancia de la existencia de la escuela, del ingreso a ella de todos los niños, de su permanencia por lo menos durante todo el ciclo 
básico. Sin embargo, al contrastar estos ideales con los resultados de los estudios sobre Promoción Automática, puede evidenciarse que la Escuela Básica Primaria colombiana, en especial la de los sectores populares, se encuentra lejos de tales propósitos. No se observa en los resultados de las investigaciones que la norma de Promoción Automática haya transformado la "cultura de la escuela tradicional" 25 ; sus concepciones de enseñar, aprender, evaluar, promocionar, afectar la disposición del espacio y el tiempo materializada en la organización de los cursos, el currículo, los períodos escolares, etc.

Una lectura desapercibida del documento podría concluir que en él se afirma que fracasó la Promoción Automática en Colombia; pero lo que podría afirmarse más bien a partir de las ideas expresadas es el fracaso del modelo escolarizador vigente, especialmente en los sectores urbano-marginales y rurales apartados, fracaso que de acuerdo con la información recolectada no se inicio con la Promoción Automática, pero que no ha podido ser contrarrestado por esta medida. Como tendencia general podría afirmarse que la escuela ha conservado, en el contexto de la Promoción Automática, sus características más cuestionadas, y sobre las cuales la norma quiso intervenir.

Lo que está aconteciendo actualmente en la escuela básica primaría no podría ser entendido por fuera del contexto sociocultural, económico y político del país. Su explicación completa haría necesaria una indagación en este sentido; sin embargo, dentro de los límites del presente artículo se presenta una hipótesis que en el marco de lo educativo podría permitir avanzar en la comprensión de la situación planteada de tal manera que puedan buscársele alternativas desde este campo específico.

El estado de la escuela actual podría explicarse por la conjunción de dos factores: de una parte, una Cultura Escolar con hondas representaciones acerca de la institución, la enseñanza, el aprendizaje, los saberes, el currículo, el maestro, los alumnos, y de otra, la ausencia de un proyecto pedagógico que pensado a largo plazo se adecue a las condiciones que reclama la educación básica de los sectores populares.

En primer lugar lo que se encuentra a partir de los diferentes estudios es que existe una manera de vivir, pensar y conceptualizar la escuela que trasciende el espacio geográfico y social. Se encuentra presente en maestros y directivos docentes independientemente de su edad, sexo, formación profesional, experiencia laboral, programa educativo en el que se encuentran, capacitación recibida. Esta manera de pensar y vivenciar la escuela pertenece a un mundo de profundas, primitivas y arraigadas representaciones simbólicas colectivas, las cuales no podrán ser afectadas por medidas puntuales, inmediatistas y sin proyecciones de largo alcance.

Por otro lado, la revisión de las políticas educativas de los últimos años que se ha presentado, pone en evidencia, que éstas se han caracterizado, de una parte, por su carácter muchas veces genérico —no adecuadas a realidades singulares, por ejemplo los sectores marginales de las metrópolis-, y de otra, por su dispersión y carácter coyuntural e inmediatista lo que las ha llevado a variar los intereses y objetivos de la escuela intentando afectarla de manera desarticulada. Lo anterior, además de dispersar la atención de maestros, directores de escuela, y responsables de la educación a nivel regional o local en múltiples problemas simultáneamente, impide afectar el mundo simbólico de lo escolar, el cual — como ya se dijo- requiere para ser transformado de esfuerzos sistemáticos, permanentes y de largo plazo.

\footnotetext{
25 "CULTURA ESCOLAR" es un concepto que se refiere a las representaciones espacio- temporales, a las ideas y modelos, a las prácticas y rituales que le sirven a maestros, alumnos, padres de familia, administradores para percibir, ordenar, relacionar e interpretar los fenómenos escolares. 
De acuerdo con lo anterior puede afirmarse que el síndrome del fracaso escolar en los sectores populares no podrá ser resuelto a menos que se ubique en la complejidad que implica y se le dé un tratamiento adecuado en magnitud, extensión y proyección. De ahí que medidas parciales y puntuales como la Promoción Automática en Colombia resulten inocuas, sino negativas y definidamente contraproducentes por las resistencias que agitan y por el grado de frustración y desesperanza que están generando respecto de las posibilidades de cambio escolar.

\section{BIBLIOGRAFIA}

AVALOS, Beatriz (1989). Enseñando a los hijos de los pobres. Un estudio etnográfico en Ame-rica Latina, Otawa, CIID.

BARCO. M. CLS (1991). Desarrollo de los procesos de Pro moción Automática en la organización y administración de la escuela en el Distrito Capital de Santafé de Bogotá. Bogotá, Universidad Externado de Colombia.

BARROS. Jesús A. (1975). Escuela Unitaria, Manual de administración y técnicas. MENICOLPE. Bogotá, septiembre.

BATISTA, E. as. (1989). Escuela y promoción escolar Medellín, Universidad de Antioquia.

BONILLA, Nohora; Cls. (1991). Evaluación de la implementación de la Promoción Automática en la educación básica primaria del departamento del Atlántico. Barranquilla, Universidad del Atlántico.

BRAVO L. SALAS, 5. (1975). Características psicopedagógicas de escolares básicos repitentes. Revista Centro de Estudios Educativos. Vol. V, No. 4.

CABALLERO, Piedad (1989). La escuela y los procesos escolares en la Promoción Automática. Bogotá, Universidad Pedagógica Nacional, 1989.

D.N.P.(Departamento Nacional de Planeación) [1991]. Plan de Apertura Educativa 19911994. Documento MEN-DNP-UDS DEC-2518. Bogotá, marzo de 1991, Mimeo.

DANE (Departamento Nacional de Estadística) (1990). Boletín No. 446. Bogotá, mayo, 1990.

DE TEZANOS, Araceli y otros (1984). Escuela y comunidad un problema de sentido. Bogotá, Ciup.

EQUIPO MEN-CIUP (1992). El mito de la escuela moderna y modernizadora. Análisis de la información cualitativa del estudio sobre repitencia escolar en Colombia. Bogotá.

EQUIPO MEN-UPN (1987a). La problemática de la evaluación escolar en Colombia Aproximación diagnóstica y propuesta de un plan para la transformación de su concepción y práctica-. Bogotá, Informe Final.

EQUIPO MEN-UPN (1988). Evaluación y cambio escolar Boletín Informativo del Plan Nacional de Evaluación. Bogotá, No. 1, junio de 1988. 
EQUIPO MEN-UPN (1987b). La problemática de la evaluación escolar en Colombia Contexto para la Promoción Automática-. Bogotá, noviembre de 1987.

FORERO, J. Cls (1991). Evaluación a la implementación de la Promoción Automática en el Caquetá. Florencia, Universidad de la Amazonía.

HADDAD, Wadi (1991). Efectos educacionales y económicos de las prácticas de reprobación y promoción en LATAPI Pablo. "Educación y escuela: Lecturas básicas para investigadores en educación”. Nueva Imagen, México, 1991.

MCGUINN-Cls. (1991). La repitencia de grado en la escuela primaria colombiana. Resultados preliminares-. Bogotá, julio de 1992. Mimeo.

Ministerio de Educación Nacional (1968). Resumen de las conferencias del profesor Alejandro Covarrubias, experto itinerante de la UNESCO sobre escuela rural. Mimeo, Bogotá.

Ministerio de Educación Nacional (1968). La Escuela Unitaria y el medio colombiano. Mimeo, Bogotá, marzo, 1963.

Ministerio de Educación Nacional (1983). Informe del tercer seminario de coordinadores de los CEP a nivel nacional, Bogotá, diciembre de 1983. Mímeo.

Ministerio de Educación Nacional (1987). Marco de referencia para la capacitación en el nuevo sistema de evaluación y Promoción Automática. Bogotá, mimeo.

MINISTERIO DE EDUCACION NACIONAL. SABER SISTEMA NACIONAL DE EVALUACION DE LA CALIDAD ESCOLAR (1992). Primeros resultados en matemáticas y lenguaje en la Escuela Básica Primaria.

MUÑOZ, C., LAVIN, S. (1988). Estrategias para mejorar el acceso a la educación primada y la permanencia en la misma. En Muñoz, C. Calidad, equidad y eficiencia de la educación primaria. CIDE, Santiago, Chile, 1988.

MYERS. R. (1978). En PROLOGO al texto SHIEFELBEIN, E. y SIMMONS, J. (1978). Los determinantes del rendimiento escolar reseña de la investigación en los países del Tercer Mundo. IDRC.

OROBIO, H. y Cls (1990). ¿Qué pasa con la Promoción Automática en Bogotá? Bogotá, Comisión Pedagógica de la Asociación Distrital de Educadores ADE, 45 pg.

ORTIZ, N. y Cls (1991). Una experiencia de formación de docentes en promoción automática y evaluación. Bogotá, Universidad de la Sabana.

PINILLA, G.; VILLEGAS, LA. (1991). Primeros resultados de la aplicación de la Promoción Automática en el departamento de Caldas. Universidad de Caldas.

POSADA, R.; PABA, C. (1991). Evaluación a la implementación de la Promoción Automática en Escuela Básica Primaria en el departamento del Magdalena. Santa Marta, Universidad del Magdalena. 
SHIEFELBEIN, E. y SIMMONS, J. (1978). Los determinantes del rendimiento escolar reseña de la investigación en los países del Tercer Mundo. IDRC.

SHIEFFELBEIN, E. (1981). Funciones de producción en educación: la experiencia en América Latina. Serie monografías, Estudios en Educación, Número 3, Washington, D.C.

SHIEFFELBEIN, E. HEIKKINEN (1991). Colombia. Acceso,, Permanencia, Repetición y eficiencia en la Educación Básica. OREALC, mimeo.

TEDESCO. Juan C. (1981). "Modelo Pedagógico y Fracaso escolar". Revista de la CEPAL.

YEPES, Antonio (1988). "La escuela como proyecto cultural". El Campesino, Bogotá, Ministerio de Educación Nacional.

YEPES, Antonio (1989). "Acción educativo-cultural aporte a la construcción de un nuevo orden social". Bogotá, Ministerio de Educación Nacional. 